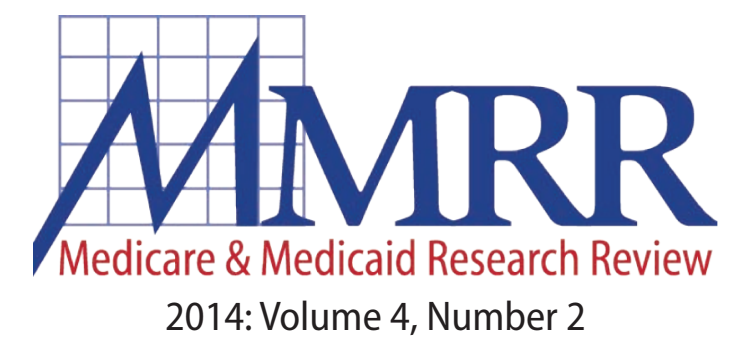

A publication of the Centers for Medicare \& Medicaid Services, Office of Information Products \& Data Analytics

\title{
Measuring Coding Intensity in the Medicare Advantage Program
}

Richard Kronick ${ }^{1}$ and W. Pete Welch ${ }^{2}$

${ }^{1}$ Department of Health and Human Services-Agency for Healthcare Research and Quality

${ }^{2}$ Department of Health and Human Services-Office of the Assistant

Secretary for Planning and Evaluation

\section{Supplement}

Article doi: $\underline{\text { http://dx.doi.org/10.5600/mmrr.004.02. }}$

$\underline{\mathrm{a} 06}$

Supplement doi: http://dx.doi.org/10.5600/

$\underline{\text { mmrr.004.02.sa06 }}$ 


\section{Medicare \& Medicaid Research Review} 2014: Volume 4, Number 2

\section{Mission Statement}

Medicare \& Medicaid Research Review is a peerreviewed, online journal reporting data and research that informs current and future directions of the Medicare, Medicaid, and Children's Health Insurance programs. The journal seeks to examine and evaluate health care coverage, quality and access to care for beneficiaries, and payment for health services.

\section{http://www.cms.gov/MMRR/}

\section{U.S. Department of Health \& Human Services Sylvia Mathews Burwell Secretary}

\section{Centers for Medicare \& Medicaid Services \\ Marilyn Tavenner \\ Administrator}

Editor-in-Chief

David M. Bott, Ph.D.

The complete list of Editorial Staff and

Editorial Board members

may be found on the MMRR Web site (click link): MMRR Editorial Staff Page

Contact: $\underline{\text { mmrr-editors@cms.hhs.gov }}$

Published by the

Centers for Medicare \& Medicaid Services.

All material in the Medicare \& Medicaid Research

Review is in the public domain and may be duplicated without permission. Citation to source is requested.

\section{Appendix}

\section{A. Decomposition of Risk Score Growth}

This section provides further information on the methods used to decompose risk score growth into the contributions of stayers, leavers, joiners, and switchers.

\subsection{4-2005 Cohort}

Between 2004 and 2005, mean risk scores grew by 0.017 for FFS and 0.042 for MA, for a differential growth of 0.025 (Exhibit A1, top panel).

\section{Decomposing Growth in the Mean Fee-for-Service (FFS) Risk Score}

Stayers:

The scores for the 30,569,191 FFS stayers increased from 1.005 in 2004 to 1.090 in 2005, an increase of 0.085 . To estimate the effects of the increase among stayers on the increase in average FFS risk score, the 0.085 increase for stayers is weighted by the stayers' share of FFS enrollment. The share is calculated relative to the average number of FFS enrollees in July, 2004 and July, 2005, which is $32,933,160$ (data not shown). The product of 0.085 and this share $(0.928)$ is 0.079 , which is the estimated contribution of FFS stayers to the change in the average FFS risk score.

Leavers, Joiners, and Switchers:

To estimate the effects of leavers, joiners, and switchers on the change in risk score, the average FFS risk score for 2004 and 2005 is subtracted from the score for each of the three groups, and then weighted by the group's share of the 2004-2005 average number of FFS enrollees. To be concrete, the average FFS risk score for 2004-2005 is 1.055 - that is, the average of the 2004 value of 1.046 and the 2005 value of 1.064 . The average 
risk score for the $1,775,685$ FFS leavers is 1.802 , or 0.747 above the two-year FFS average. The FFS leavers account for $5.4 \%$ of the average number of FFS enrollees in 2004 and 2005. Multiplying 0.747 by 0.054 yields 0.040 - that is, FFS risk scores in 2005 are 0.040 lower than they would have been if there had been no FFS leavers or if people who did leave had risk scores that averaged 1.055 in both 2004 and 2005.

Based on similar calculations, the $2,267,782$ FFS joiners lowered 2005 FFS risk scores by 0.025 . The 534,225 switchers from FFS to MA increased the average FFS risk score slightly (by 0.003 , because switchers to MA had lower than average risk scores, so their departure from FFS led to slight increases in the 2005 scores). And the 150,246 switchers from MA to FFS had virtually no effect on FFS scores, primarily because there were so few of them.

Average risk scores for FFS stayers increased substantially (by 0.085 ) from 2004 to 2005, but the average risk score for all FFS enrollees increased by only 0.017 . As shown in Exhibit A1, 'caseload dynamics' account for the difference-high risk score decedents exited the caseload, being replaced by low risk score joiners.

\section{Comparison of Components of MA and FFS Risk Score Growth}

As shown in Exhibit A1, average MA risk scores from 2004 to 2005 increased by 0.042 , or 0.025 more than the FFS increase of 0.017 . The more rapid increase in MA average risk occurred not only because risk scores increased faster for MA stayers than for FFS stayers, but also because caseload dynamics restrained MA risk score growth more strongly than they restrained FFS risk score growth. (See right-most column for net contributions.) The stayer share of MA enrollment is 88\%: 4,518,275 MA stayers divided by the average 2004 and 2005 MA enrollment $(5,114,195$, data not shown). The product of this $88 \%$ and the 0.104 increase in MA risk scores yields 0.092 , the effect of stayers on MA score growth. The difference between
MA and FFS in the effects of stayers on 2005 risk score is $0.013(0.092-0.079)$, which represents the contribution of stayers to differential growth of MA and FFS for the 2004-2005 cohort (see Exhibit 3).

The 228,787 MA leavers account for $4.5 \%$ of the 2004-2005 average MA enrollment, substantially less than the $5.4 \%$ accounted for by FFS leavers (data not shown). Further, the difference between the 1.802 average score of FFS leavers and the 1.055 FFS two-year average is larger than the difference between the 1.657 score for MA leavers and the 0.965 MA average; that is, 0.747 vs. 0.692 . Similarly, MA joiners account for $5.4 \%$ of average enrollment, compared to $6.9 \%$ for FFS joiners. Largely, as a result of the fact that MA joiners and leavers are a smaller share of enrollment than FFS joiners and leavers, the effects of caseload dynamics on restraining yearover-year growth in scores are substantially weaker in MA than in FFS-0.009 weaker for leavers, and 0.008 weaker for joiners. These differences are partially balanced by the effects of switchers, but even when switchers are considered, caseload dynamics caused MA average risk scores from 2004 to 2005 to increase by 0.012 more quickly than average FFS risk scores.

\section{2012-2013 Cohort Compared to 2004-2005 Cohort}

As shown in the bottom panel of Exhibit A1, the effects of caseload dynamics reversed in 20122013, slowing MA scores' relative growth. The main changes in caseload dynamics are in the effects of joiners and switchers. As in 2004-2005, joiners are a smaller share of average MA enrollment than of average FFS enrollment $(7.0 \%$ in MA compared to $8.2 \%$ in FFS, data not shown), but the difference between the average score for joiners and the two-year average is larger (in absolute value) for MA than for FFS (in FFS, 0.705-1.157 = -0.452, compared to MA, $0.692-1.227=-0.535)$. The lower prevalence of MA joiners compared to FFS 
Exhibit A1. Decomposition of the Change in MA and FFS Risk Scores, 2004-2005 and 2012-2013

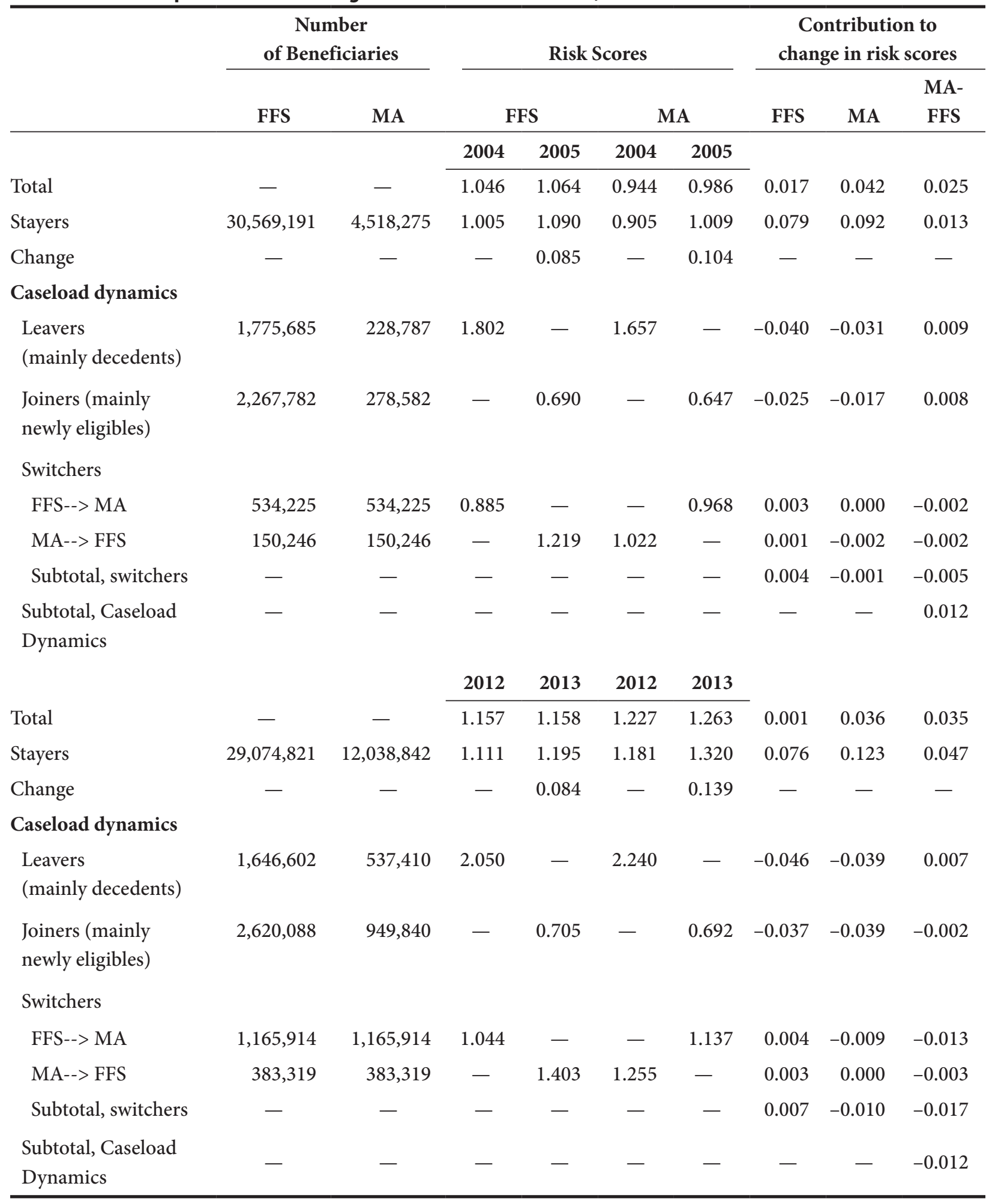

SOURCE:Authors' analysis of Medicare beneficiary-level administrative data.

joiners is largely balanced by the larger difference between the sectors in average risk scores and, as a result, the effects of MA joiners on the 2012-2013 change in MA average score is -0.039 , which is not 
much different from the -0.037 estimate for FFS. In 2004-2005, joiners restrained FFS scores by 0.008 more than MA, but in 2012-2013 the effect was only 0.002 .

There is an even larger difference between 2004 to 2005 and 2012 to 2013 in the effects of switchers on relative MA and FFS scores. Of the FFS enrollees in July, 2012, 1,165,914 switched to MA by July, 2013. These beneficiaries had lowerthan-average FFS scores in 2011, so their departure from FFS caused the 2012 FFS average score to increase. They also had lower scores than the MA average, so their entrance into MA lowered the average 2013 MA score. And while the effects are relatively small, the 383,319 switchers from MA to FFS slightly amplified this trend-their 2012 score was very close to the MA average, so their departure from MA had little effect on the 2012 average MA score. However, their 2013 score of 1.403 was much higher than the FFS average and, thus, contributed to an increase in the average 2013 FFS score. On net, switchers in 2012-2013 lowered MA scores relative to FFS by 0.017 more slowly than FFS scores, larger than their 0.005 effect in 2004-2005.

To summarize the 2012-2013 findings, the average MA score increased by 0.036 , or 0.035 more quickly than the average FFS increase of 0.001 . However, the effect of stayers was to increase the average MA score by 0.047 more quickly than the average FFS score. The fact that the average MA score increased by 'only' 0.035 more quickly was a result of caseload dynamics-primarily the effects of switchers in restraining MA score growth.

\section{Decomposition Across Nine Cohorts}

Exhibit A2 shows similar results for each cohort from 2004 to 2005 through 2012 to 2013, and demonstrates that over this time period, caseload dynamics had minimal net effect

Exhibit A2. Decomposition of the Differential Change in MA and FFS Risk Scores, By Cohort

\begin{tabular}{|c|c|c|c|c|c|c|c|c|c|c|}
\hline & \multicolumn{10}{|c|}{ Cohort } \\
\hline & 2004-05 & $2005-06$ & 2006-07 & 2007-08 & 2008-09 & 2009-10 & 2010-11 & 2011-12 & 2012-13 & Average \\
\hline Total, A & 0.025 & 0.015 & 0.024 & 0.026 & 0.012 & 0.014 & 0.027 & 0.014 & 0.035 & 0.021 \\
\hline \multicolumn{11}{|c|}{ Contribution to change in risk score } \\
\hline Stayers, B & 0.013 & 0.006 & 0.016 & 0.027 & 0.026 & 0.022 & 0.033 & 0.027 & 0.047 & 0.024 \\
\hline \multicolumn{11}{|c|}{ Caseload dynamics } \\
\hline Leavers, C & 0.009 & 0.012 & 0.011 & 0.009 & 0.007 & 0.005 & 0.006 & 0.005 & 0.007 & 0.008 \\
\hline Joiners, D & 0.008 & 0.002 & 0.002 & -0.001 & -0.001 & 0.001 & 0.002 & -0.001 & -0.002 & 0.001 \\
\hline \multicolumn{11}{|l|}{ Switchers } \\
\hline FFS--> MA & -0.002 & -0.002 & 0.000 & -0.004 & -0.014 & -0.012 & -0.012 & -0.014 & -0.013 & -0.008 \\
\hline MA--> FFS & -0.002 & -0.004 & -0.004 & -0.005 & -0.005 & -0.002 & -0.002 & -0.003 & -0.003 & -0.003 \\
\hline Subtotal, E & -0.005 & -0.006 & -0.004 & -0.009 & -0.020 & -0.014 & -0.014 & -0.017 & -0.017 & -0.012 \\
\hline Total, $\mathrm{F}=\mathrm{C}+\mathrm{D}+\mathrm{E}$ & 0.012 & 0.008 & 0.009 & 0.000 & -0.013 & -0.008 & -0.006 & -0.013 & -0.012 & -0.003 \\
\hline Residual, A-B-F & 0.000 & 0.000 & 0.000 & -0.001 & 0.000 & 0.000 & 0.000 & 0.000 & 0.000 & 0.000 \\
\hline
\end{tabular}


on the differential growth between MA and FFS in average risk scores. ${ }^{1}$ In the first three cohorts-2004-2005, 2005-2006, and 20062007, caseload dynamics caused the average MA score to increase more quickly than the average FFS score-by $0.012,0.008$, and 0.009 in the three cohorts respectively. In 2007-2008, the estimated net effect of caseload dynamics was zero-the differential effect of leavers in increasing MA score growth was balanced by the effects of switchers in restraining it. And in each of the last five cohorts-2008-2009, 2009-2010, 20102011, 2011-2012, and 2012-2013-differential caseload dynamics caused MA scores to increase more slowly than FFS, by $-0.013,-0.008,-0.006$, -0.013 , and -0.012 respectively. Combining the results across all nine cohorts, there is minimal net effect of differential caseload dynamics-the first three cohorts are largely balanced by effects in the last five cohorts.

\section{B. Contract-Level Coding Intensity}

This section describes the contract-level methodology used to create Exhibit 8.

HCC risk scores are composed of demographic and diagnostic components. Because only the diagnostic component can be affected by coding intensity, we exclude risk score changes that result from changes in demographic characteristics.

The contract-level results analyze MA stayers, defined as beneficiaries who were enrolled in

\footnotetext{
${ }^{1}$ With the exception of 2007-2008, the numbers in the top row of Exhibit A2 are identical to the numbers used in Exhibit 1. In the analysis for Exhibit A2, diagnoses from diagnostic radiology claims were excluded in the 2008 risk scores, based on diagnostic information from 2007. This exclusion was made because health plans were instructed to exclude diagnostic radiology claims as a source of information for diagnoses for dates of service in 2007 and subsequent years on the theory that many diagnoses on radiology claims are 'rule out' diagnoses and should not be included. As a result, the average FFS risk score decreased from 2007 to 2008 in Exhibit 1. For the sake of consistency, we include diagnostic radiology as a source of diagnoses in the 2007-2008 cohort, but excluded it in the 2008-2009 and subsequent cohorts.
}

the same contract in July of two successive years and whose diagnostic data also were provided by that contract. For example, to be included in the 2004-2005 stayer cohort for a given contract, an enrollee must have been enrolled in that contract in July 2004 and July 2005, and must have been enrolled in that contract for all of calendar year 2003 and calendar year 2004.

Because risk scores among FFS stayers increase more quickly for older than younger beneficiaries and increase sharply in the last year of life, we adjust the change in the FFS score for age and survivor status using direct standardization, using five-year age groups, and defining survivors as enrollees still alive on December 31 of the second year in the cohort. This direct standardization is performed for each contract and cohort. For each contract, the difference between the MA score and this standardized FFS score is calculated for each cohort. The sum of this difference across the seven cohorts is called the 'cumulative excess increase in risk scores among stayers.'2

The cumulative excess increase for all enrollees is calculated in two steps. ${ }^{3}$ First, the increase in risk score is calculated as a contract's 2011 risk score minus its 2004 risk score. Second, we subtract the increase in the FFS risk from 2004 to 2011 (0.099) from the contract-specific 2004-2011 increase, yielding the contract-level cumulative excess increase in risk score from 2004 to 2011.

Our main purposes in this analysis are to measure the heterogeneity across contracts in the cumulative excess increase in risk scores among stayers and to investigate the extent to which a measure free of the impact of changes of enrollment mix (cumulative excess increase

\footnotetext{
${ }^{2}$ Data for 2012 and 2013 became available after the contractlevel analysis was conducted, and in the interest of not delaying publication of this paper, we have not updated this analysis with the 2012 and 2013 data.

${ }^{3}$ Demographic HCCs are included in the all-enrollee score but not the stayer score.
} 
for stayers) predicts the comprehensive measure (cumulative excess increase for the contact as a whole). We restrict the analysis to contracts that had at least 10,000 enrollees in 2004 and 2011 and whose 2011 enrollment was between $25 \%$ and $300 \%$ of its 2004 enrollment, because the change in risk scores for those excluded contracts is likely strongly affected by changes in the composition of enrollees. The 86 contracts meeting these criteria accounted for 42 percent of MA enrollment in 2011.

\section{Change in Mortality Rates by Sector}

We estimate the mortality rate for FFS and MA enrollees for each year from 2004 to 2012 as follows. The denominator is the number of beneficiaries enrolled and alive on January 1 of a given year; the numerator includes the subset of those beneficiaries who died on or before December 31 of that year. As shown in Exhibits A3 and A4, crude mortality rates were lower in MA than FFS in 2004-0.0411 in MA compared to 0.0473 in FFS. Mortality declined in both sectors between 2004 and 2012, but declined more rapidly in MA-by $1.5 \%$ per year in MA, compared to $0.7 \%$ per year in FFS. The relative mortality rate in MA declined from $87 \%$ in 2004 to $81 \%$ in 2012 .

Part of the reason that relative mortality declined in MA compared to FFS is that the surge in MA enrollment post-2006 brought more relatively young beneficiaries into MA, reducing the average age of MA enrollees and resulting in lower mortality rates. We apply an age-sex adjustment to mortality rates using the indirect method, with FFS in 2004 as the reference category. Controlling for age and gender, mortality in MA declined at approximately the same rate as in FFS from 2004-2012 (not shown), although even on an unadjusted basis, the decline in mortality was somewhat more rapid in MA than in FFS.

Exhibit A3. Mortality Rate, MA vs. FFS, 2004-2012

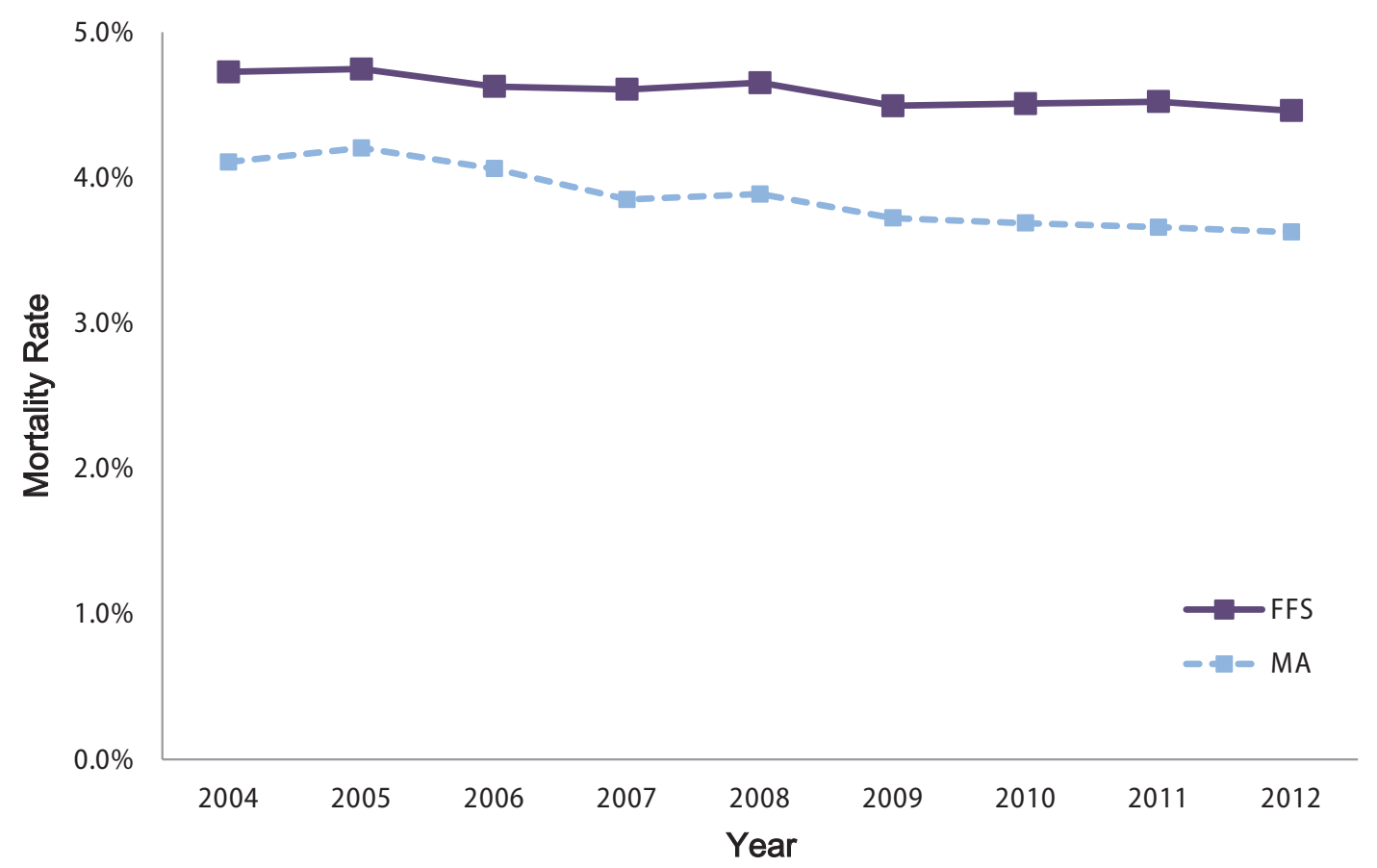

SOURCE: Authors' analysis of Medicare administrative data. 
Exhibit A4. Mortality Rate, FFS vs. MA, by Year

\begin{tabular}{lccc}
\hline Year & FFS & MA & MA-FFS \\
\hline 2004 & 0.0473 & 0.0411 & $87 \%$ \\
2005 & 0.0475 & 0.0420 & $89 \%$ \\
2006 & 0.0463 & 0.0406 & $88 \%$ \\
2007 & 0.0461 & 0.0385 & $84 \%$ \\
2008 & 0.0465 & 0.0389 & $84 \%$ \\
2009 & 0.0449 & 0.0372 & $83 \%$ \\
2010 & 0.0451 & 0.0369 & $82 \%$ \\
2011 & 0.0452 & 0.0366 & $81 \%$ \\
2012 & 0.0446 & 0.0363 & $81 \%$ \\
annualized change, $2004-12$ & \\
$\%$ & $-0.7 \%$ & $-1.5 \%$ & $-0.8 \%$ \\
\multicolumn{2}{l}{ SOURCE: Authors' analysis of Medicare beneficiary level } \\
administrative data.
\end{tabular}

If MA enrollees were actually increasing in morbidity relative to FFS enrollees, we would expect that relative mortality rates in MA would also increase. The data in Exhibit A4 provide no support for the hypothesis of increasing relative morbidity of MA enrollees.

\section{Relative Prevalence by HCC}

Extending the results from Exhibit 7 in the article, Exhibit A5 presents the prevalence of each HCC in FFS, MA, and among the top decile of plans in terms of coding intensity. For HCCs in which coding is relatively non-discretionary, such as acute myocardial infarction (HCC 81, ranked 34 out of 70 HCCs) and hip fracture (HCC 158, ranked 49), prevalence in the top decile of plans is similar to or less than FFS prevalence, suggesting that there is little difference in underlying morbidity.

\section{E. Relative Cost per Enrollee as Predicted Using MCBS Data}

CMS annually conducts the Medicare Current Beneficiary Survey (MCBS), surveying both FFS and MA enrollees. MCBS collects data for both sets of beneficiaries on overall health status and on self-report of certain chronic disease. Data for beneficiaries in the two sectors were collected through the same mechanism.

As shown in Exhibit A6, the self-reported prevalence of diabetes is slightly higher among MA enrollees than among FFS beneficiaries, but self-reported prevalence of cancer and heart failure is lower in MA than in FFS. Self-reported health status is similar in the two groups (data not shown). However, MA enrollees are much less likely than FFS beneficiaries to be institutionalized $(2.1 \%$ compared to $4.9 \%$ ), and being institutionalized is associated with a large increase in expenditures, even after controlling for other factors.

We used this database to estimate the relative cost of FFS and MA enrollees. In the first stage, we regressed FFS cost on variables, such as health status, using only FFS enrollees. In the second stage, we used the regression results to predicted cost for both FFS and MA enrollees.

In the first stage, beneficiaries were excluded if they were enrolled in MA plans, did not have both Parts $A$ and $B$, were in the ESRD program, or were in hospice. Pooling these data across the seven years of 2005-2011, we predicted Medicare cost (Parts A and B) for both FFS and MA enrollees. The regression had 63,803 observations (about 9,000 per year).

As shown in Exhibit A7, the regression had an $\mathrm{R}$-square of 0.1047 . The variables with the largest coefficients were self-reported health status and a marker for institutionalization. Were a beneficiary's health to fall from excellent to poor, his/her expected cost would increase by $\$ 11,522$, which is $151 \%$ of the mean of the dependent variable, namely $\$ 7,630$. Institutionalized beneficiaries had expected costs $\$ 8,742$ higher than beneficiaries living in the community, which is $115 \%$ of the mean cost. Both variables are highly significant. Except for hypertension, which is insignificant, all the medical condition variables, which come from a question about whether the respondent has ever 


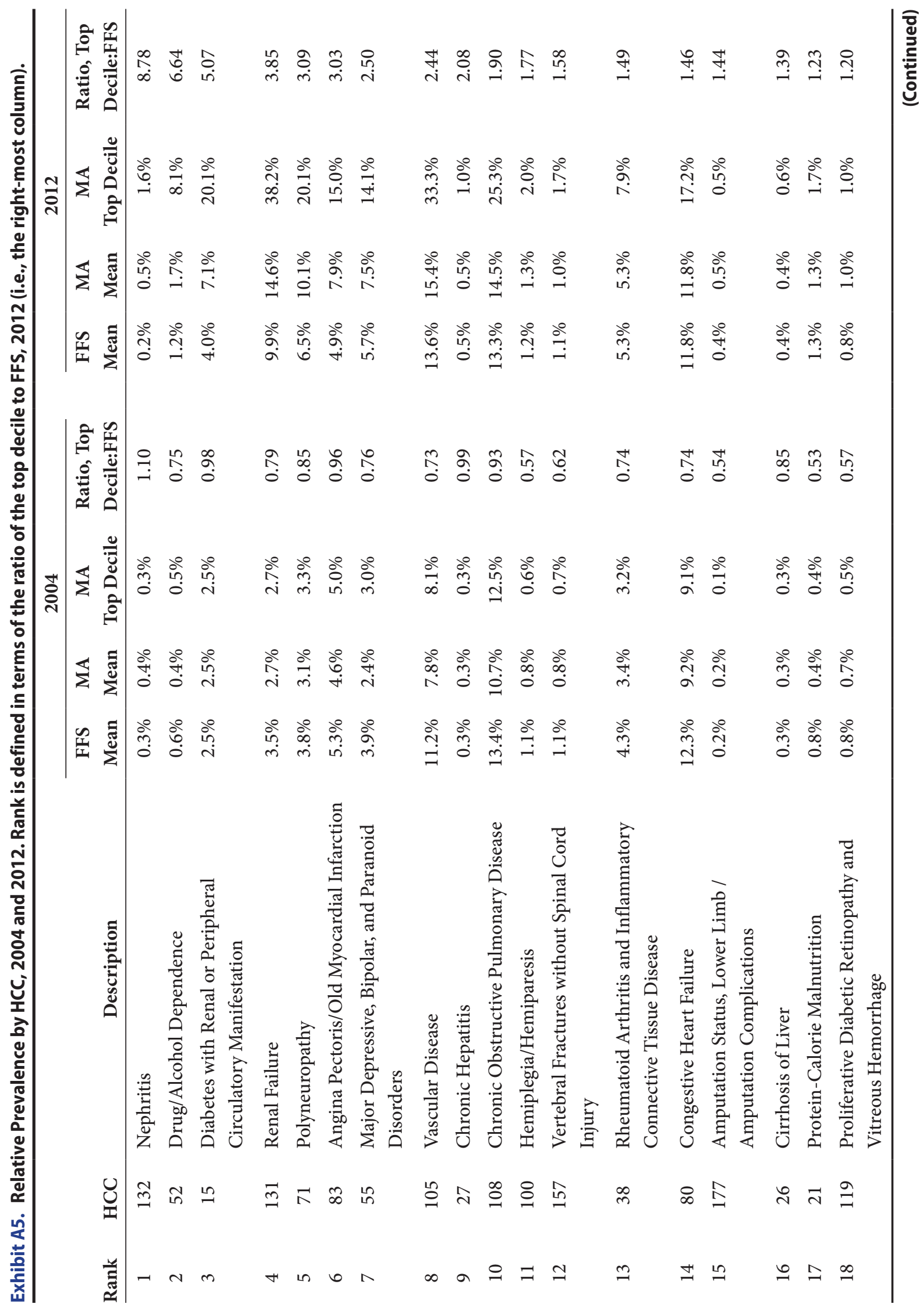




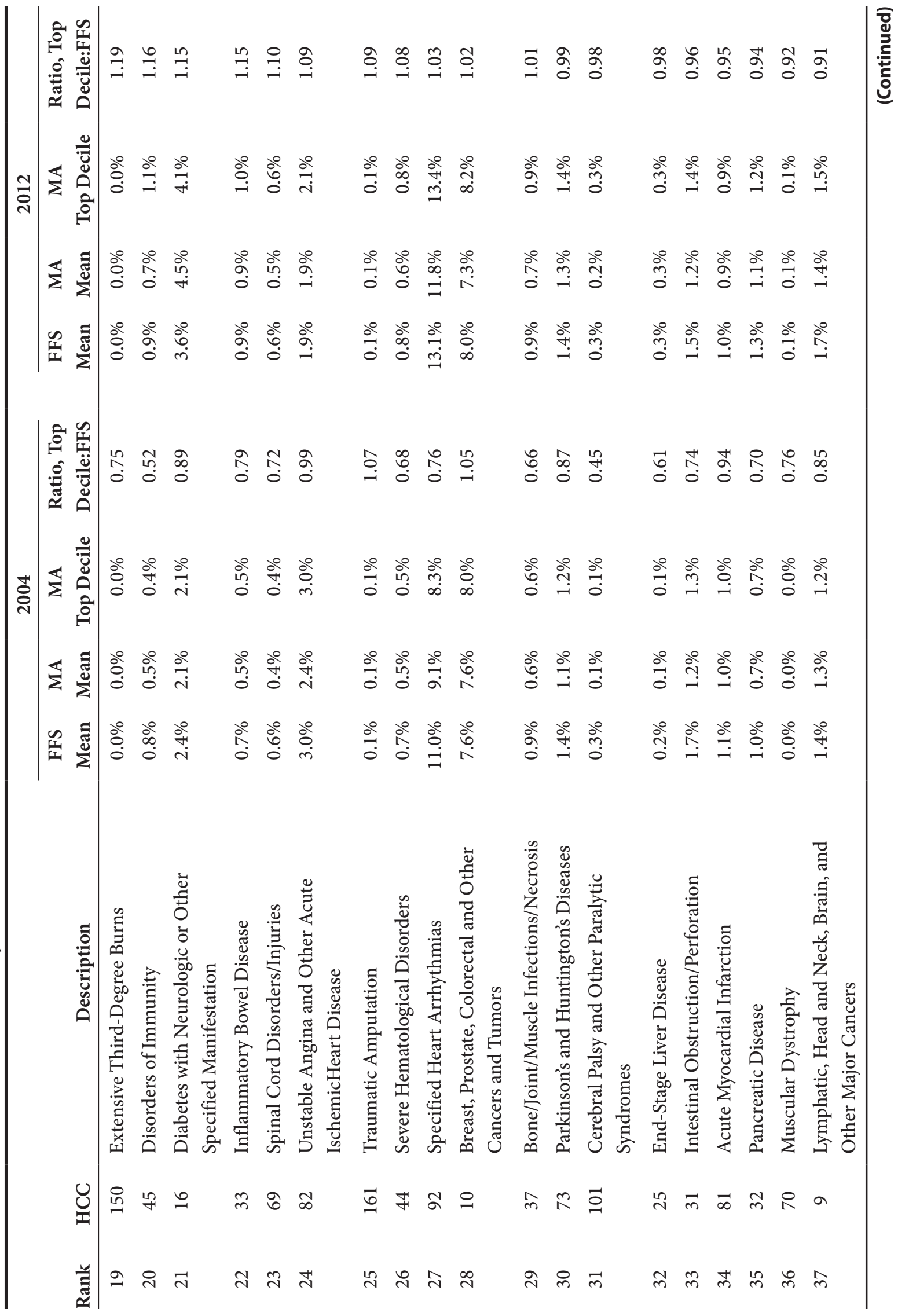




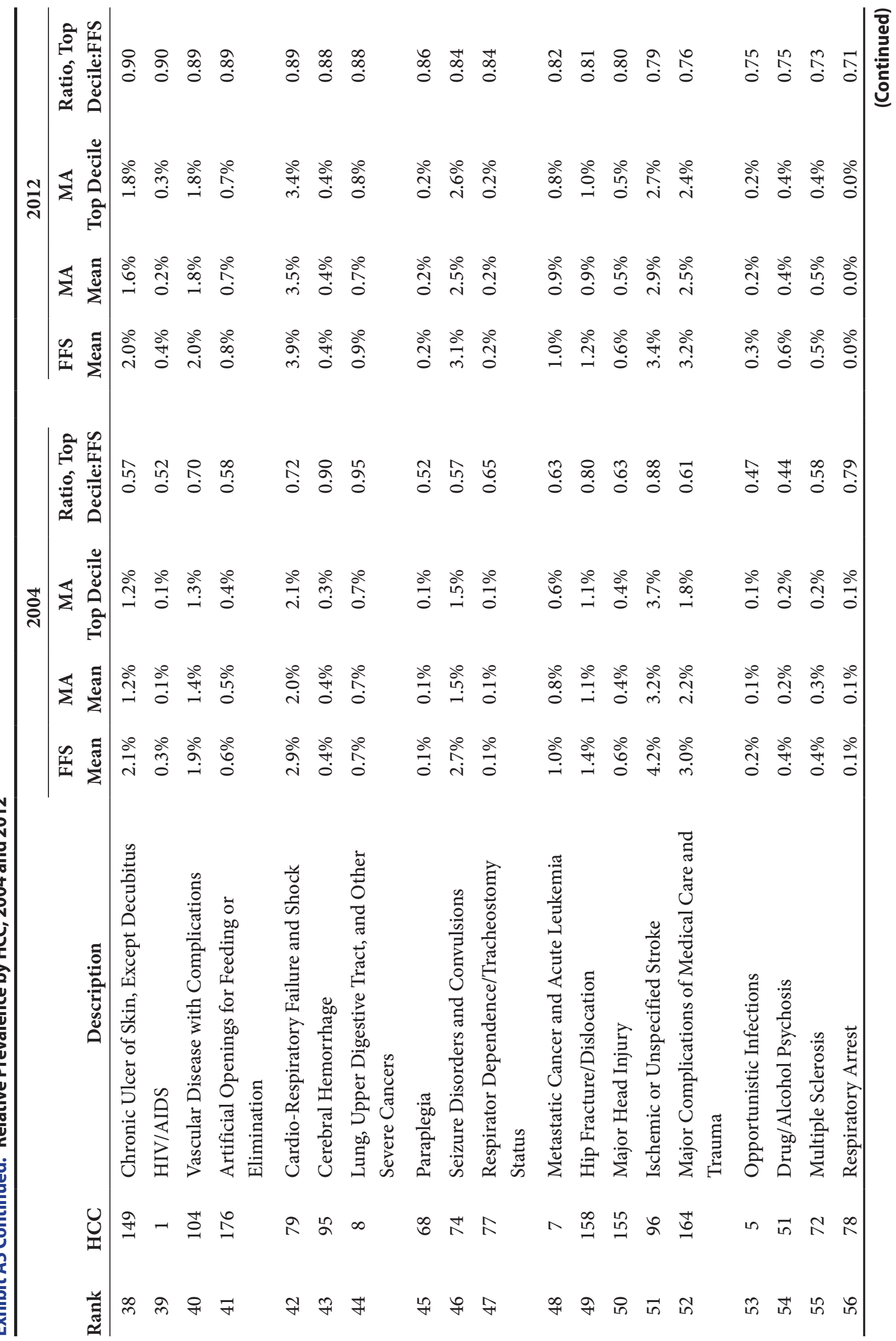




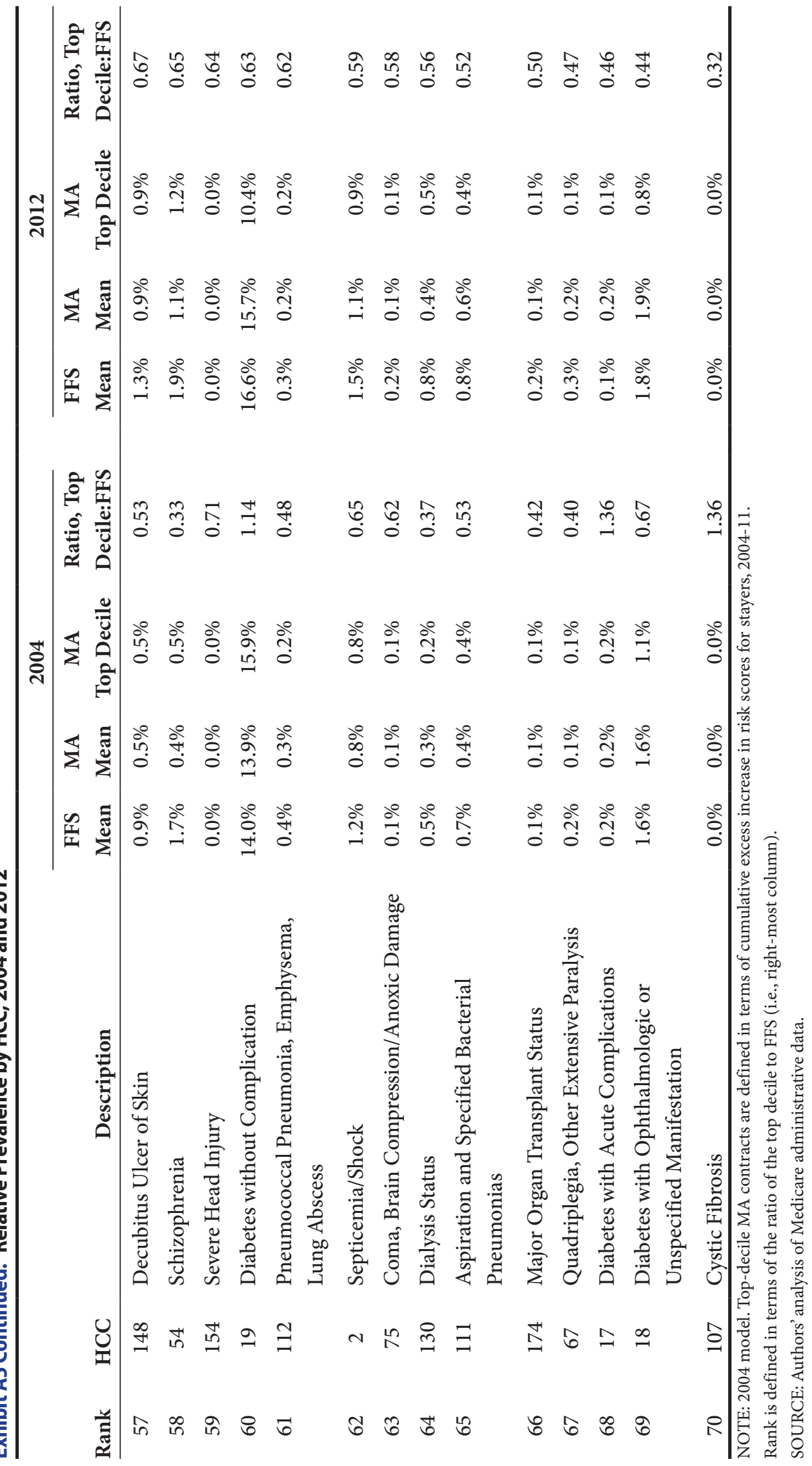


Exhibit A6. Prevalence of Selected Chronic Conditions, MCBS, MA vs. FFS, 2006-2011 Average

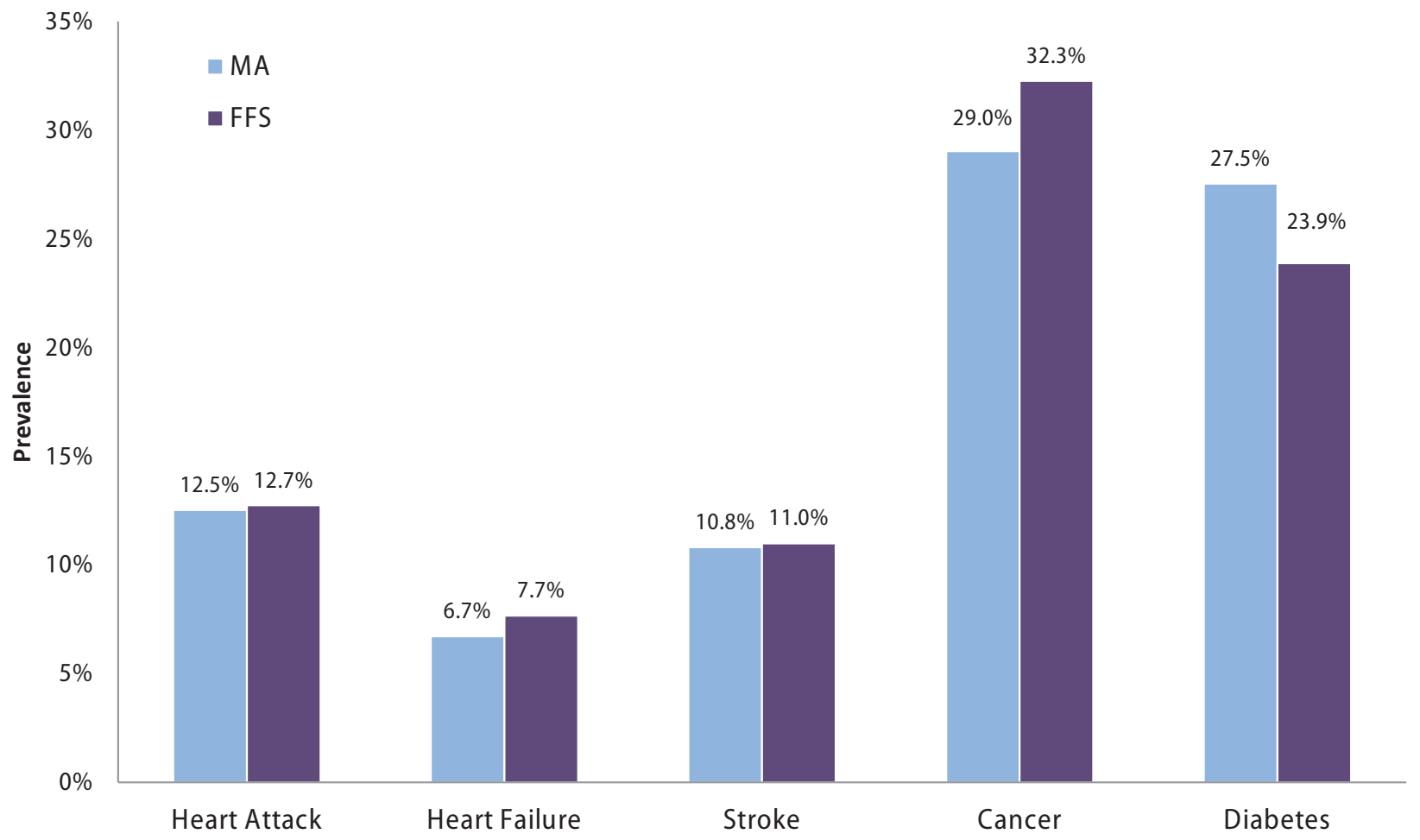

NOTE: Self-reported of ever having been diagnosed with selected chronic condition.

Simple average of prevalence for each year.

SOURCE: Authors' analysis of Medicare Current Beneficiary Survey (MCBS) data.

been told that she had a specified condition, are highly significant and in the expected direction with coefficients ranging between $\$ 1,338$ (diabetes) and $\$ 5,521$ (heart failure).

In the second stage, expenditures for each beneficiary (whether in MA or FFS) are predicted using this equation. That is, although we do not know the actual expenditures for MA enrollees, we do know whether they had diabetes and heart failure, for instance. Exhibit A8 reports the ratio of the mean expenditure for MA enrollees and the mean for FFS enrollees. ${ }^{4}$

${ }^{4}$ MCBS was also used by J. M. McWilliams, J. Hsu, \& J. P. Newhouse. (2012, December). New Risk-Adjustment System Was Associated With Reduced Favorable Selection in Medicare Advantage. Health Affairs, 31(12),2630-2640. However, they did not use health status measures to predict relative expenditures. They did report health status (e.g., fair or poor health) and a summary measure of actual utilization (their Exhibit 4).

\section{F. Bid as a Percentage of Benchmark as Metric of Overpayment}

MA plans submit bids defined in terms of PMPM (per member per month) payment and standardized for an enrollee with a risk score of 1.0. Medicare calculates benchmarks (also defined in terms of PMPM for a risk score of 1.0) at the county level. If a plan's bid equals or exceeds its benchmark, the plan is paid the benchmark. If the bid is less than its benchmark, the plan is paid its bid plus a rebate. In 2010 (the year of our data), the rebate was $75 \%$ of the differential between its benchmark and its bid. The rebate is defined in terms of PMPM, but is not standardized for the risk score. Rebates must be used to benefit enrollees in the form of supplemental benefits or lower premiums. 
Exhibit A7. Regression of Cost on Diagnoses and Other Variables from MCBS (Fee-for-Service Enrollees Only)

\begin{tabular}{|c|c|c|}
\hline Variable & Coefficient & t Value \\
\hline \multicolumn{3}{|c|}{ Health status and diagnoses } \\
\hline Health status & 11,522 & 47.98 \\
\hline Heart attack & 1,980 & 10.00 \\
\hline Heart failure & 5,521 & 22.43 \\
\hline Stroke & 1,848 & 9.13 \\
\hline Cancer & 2,145 & 15.66 \\
\hline Diabetes & 1,338 & 8.81 \\
\hline Hypertension & 31 & 0.23 \\
\hline \multicolumn{3}{|l|}{ Status } \\
\hline Medicaid & 1,365 & 7.44 \\
\hline Institutionalized & 8,742 & 28.24 \\
\hline \multicolumn{3}{|l|}{ Age (female) } \\
\hline$<35$ & $-1,204$ & -1.50 \\
\hline $35-44$ & $-1,913$ & -3.04 \\
\hline $45-54$ & $-2,758$ & -5.72 \\
\hline $55-64$ & $-1,469$ & -3.71 \\
\hline $65-69$ & 319 & 1.16 \\
\hline $70-74$ & 732 & 2.68 \\
\hline $75-79$ & 1,711 & 5.98 \\
\hline $80-84$ & 2,055 & 6.92 \\
\hline$>84$ & 1,837 & 6.30 \\
\hline \multicolumn{3}{|l|}{ Age (male) } \\
\hline$<35$ & $-2,074$ & -2.78 \\
\hline $35-44$ & $-2,350$ & -3.94 \\
\hline $45-54$ & $-1,019$ & -2.32 \\
\hline $55-64$ & $-2,906$ & -7.57 \\
\hline \multicolumn{3}{|l|}{$\begin{array}{l}65-69 \text { (reference } \\
\text { category) }\end{array}$} \\
\hline $70-74$ & 498 & 1.73 \\
\hline $75-79$ & 2,106 & 6.88 \\
\hline $80-84$ & 2,105 & 6.30 \\
\hline$>84$ & 2,590 & 7.16 \\
\hline \multicolumn{3}{|l|}{ Year } \\
\hline \multicolumn{3}{|l|}{$\begin{array}{l}2006 \text { (reference } \\
\text { category) }\end{array}$} \\
\hline 2007 & 148 & 0.7 \\
\hline 2008 & 233 & 1.1 \\
\hline 2009 & 894 & 4.17 \\
\hline
\end{tabular}

Exhibit A7 Continued. Regression of Cost on Diagnoses and Other Variables from MCBS (Fee-for-Service Enrollees Only)

\begin{tabular}{lcc}
\hline Variable & Coefficient & t Value \\
\hline 2010 & 1,522 & 7.11 \\
2011 & 1,749 & 8.16 \\
Intercept & $-1,598$ & -5.94 \\
\hline NOTES: ${ }^{*}$ p $<.001$; R-square: $0.1047 ;$ F value: $240.45 \mathrm{p}<.0001 ;$ \\
N= 63,802; Health Status is coded as follows: Excellent=1; Very \\
good=0.75; Good=0.5; Fair=0.25; Poor=0. All other variables in the \\
model are 0-1 indicator variables. \\
SOURCE: Authors' analysis of Medicare Current Beneficiary Survey \\
(MCBS) data.
\end{tabular}

An Excel file with plan-level data for 2010 was downloaded from the CMS Web site. ${ }^{5}$ Its four key variables were plan ID, "payment," and "rebate" (both defined in terms of PMPM), and "score." As long as the rebate was not zero, algebraically the bid and the benchmark can be calculated. ${ }^{6}$

As shown in Exhibit A9, the mean bid percentage calculated by decile ranged from $84 \%$ to $93 \%$ for the first seven deciles, was $81 \%$ for the eighth decile, was $78 \%$ for the ninth decile, and fell sharply to $60 \%$ for the tenth decile. For deciles one through seven, there was relatively little relationship between coding intensity and bid as a percentage of the benchmark. However, this percentage was lower for the contracts in the eighth and ninth decile and was much lower for the contracts with the highest coding intensity.

The markedly lower bids as a percentage of benchmarks for contracts with rapidly increasing risk scores for stayers strengthen the argument that a policy response targeted on contracts with high coding intensity will not prove overly disruptive to the MA market. In response, those contracts would likely increase their bids, they would receive smaller rebates, and the value of extra benefits to beneficiaries would be reduced somewhat.

\footnotetext{
${ }^{5}$ http://cms.gov/Medicare/Medicare-Advantage/Plan-Payment/ Plan-Payment-Data.html (assessed early April 2013). We used the most current data then available.

${ }^{6}$ Although the variables were initially at the plan level, weighting by enrollment we calculated the means of payment, rebate, and score prior to calculating bid and benchmark at the contract level.
} 
Exhibit A8. Predicted Cost per Enrollee, MA as a Percentage of FFS, MCBS, 2006-2011

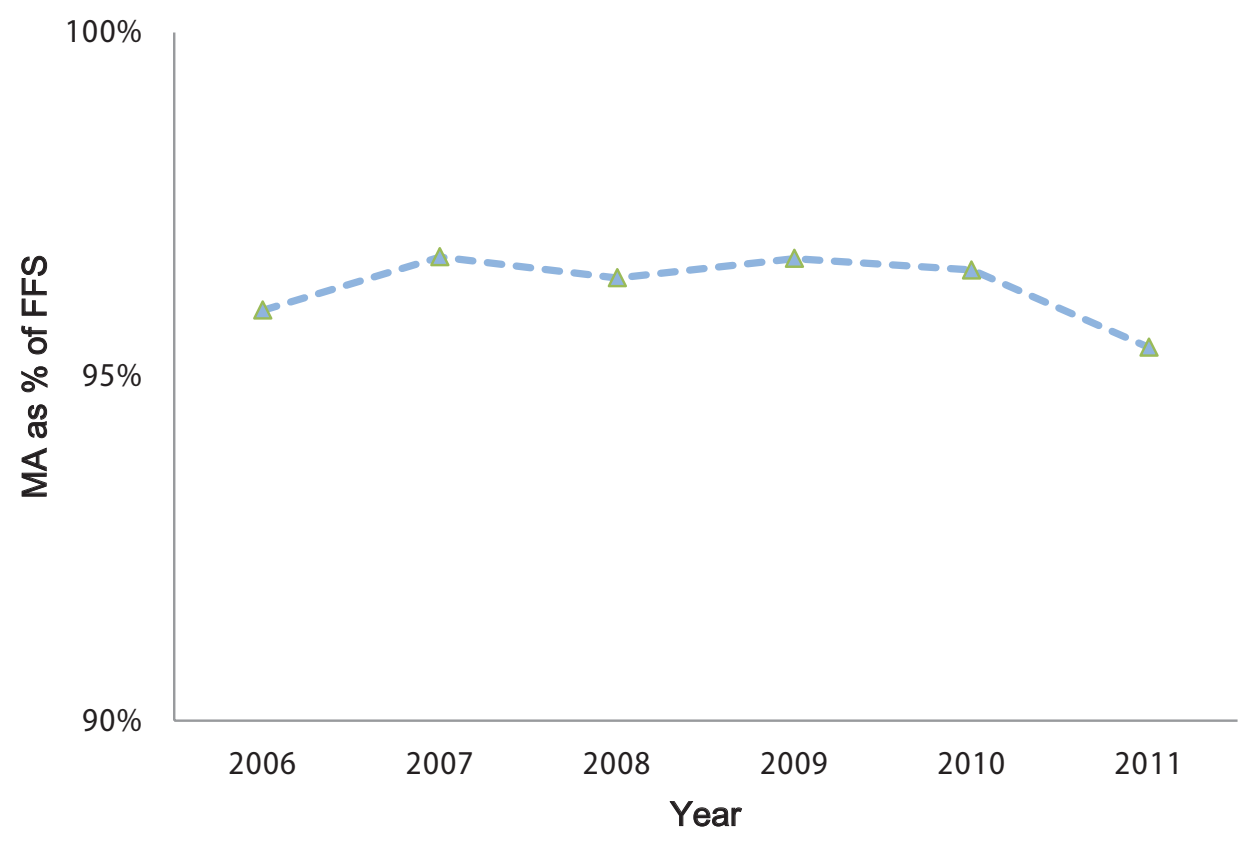

SOURCE: Authors' analysis of Medicare Current Beneficiary Survey (MCBS) data.

Exhibit A9. Bid as a Percentage of Benchmark by Decile of Excess Increase in Risk Score Among Stayers, 2010

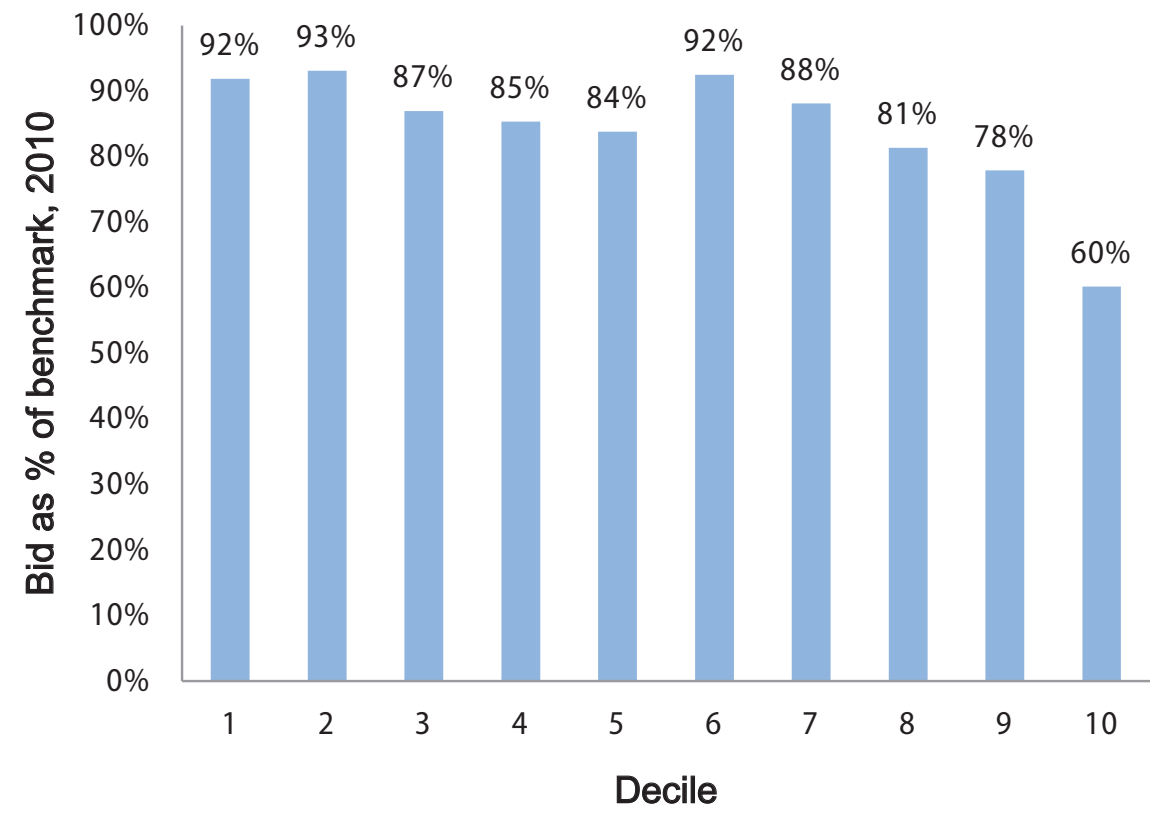

NOTE: Continuously participating contracts $(\mathrm{N}=86)$.

SOURCE: Authors' analysis of Medicare Current Beneficiary Survey (MCBS) data. 